\title{
Light Dark Matter Search with Ionization Signals in XENON1T
}

E. Aprile, ${ }^{1}$ J. Aalbers $\odot,{ }^{2, *}$ F. Agostini, ${ }^{3}$ M. Alfonsi, ${ }^{4}$ L. Althueser,${ }^{5}$ F. D. Amaro, ${ }^{6}$ V. C. Antochi, ${ }^{2}$ E. Angelino, ${ }^{7}$ F. Arneodo, ${ }^{8}$ D. Barge,${ }^{2}$ L. Baudis, ${ }^{9}$ B. Bauermeister, ${ }^{2}$ L. Bellagamba, ${ }^{3}$ M. L. Benabderrahmane, ${ }^{8}$ T. Berger,${ }^{10}$ P. A. Breur, ${ }^{11}$ A. Brown, ${ }^{9}$ E. Brown, ${ }^{10}$ S. Bruenner, ${ }^{12}$ G. Bruno, ${ }^{8}$ R. Budnik, ${ }^{13}$ C. Capelli, ${ }^{9}$ J. M. R. Cardoso, ${ }^{6}$ D. Cichon, ${ }^{12}$ D. Coderre, ${ }^{14}$ A. P. Colijn, ${ }^{11, \dagger}$ J. Conrad, ${ }^{2}$ J. P. Cussonneau ${ }^{15}$ M. P. Decowski, ${ }^{11}$ P. de Perio, ${ }^{1}$ A. Depoian, ${ }^{16}$ P. Di Gangi, ${ }^{3}$ A. Di Giovanni, ${ }^{8}$ S. Diglio, ${ }^{15}$ A. Elykov, ${ }^{14}$ G. Eurin, ${ }^{12}$ J. Fei, ${ }^{17}$ A. D. Ferella, ${ }^{2}$ A. Fieguth, ${ }^{5}$ W. Fulgione, ${ }^{18,7}$ P. Gaemers, ${ }^{11}$ A. Gallo Rosso, ${ }^{18}$ M. Galloway, ${ }^{9}$ F. Gao ${ }^{1}$ M. Garbini, ${ }^{3}$ L. Grandi, ${ }^{19}$ Z. Greene, ${ }^{1}$ C. Hasterok, ${ }^{12}$ C. Hils, ${ }^{4}$ E. Hogenbirk, ${ }^{11}$ J. Howlett, ${ }^{1}$ M. Iacovacci, ${ }^{20}$ R. Itay, ${ }^{13}$ F. Joerg, ${ }^{12}$ S. Kazama, ${ }^{21}$ A. Kish, ${ }^{9}$ M. Kobayashi, ${ }^{1}$ G. Koltman, ${ }^{13}$ A. Kopec, ${ }^{16}$ H. Landsman, ${ }^{13}$ R. F. Lang, ${ }^{16}$ L. Levinson, ${ }^{13}$ Q. Lin, ${ }^{1}$ S. Lindemann, ${ }^{14}$ M. Lindner, ${ }^{12}$ F. Lombardi, ${ }^{6,17}$ J. A. M. Lopes, ${ }^{6 »}$ E. López Fune, ${ }^{22}$ C. Macolino, ${ }^{23}$ J. Mahlstedt, ${ }^{2}$ A. Manfredini, ${ }^{9,13}$ F. Marignetti, ${ }^{20}$ T. Marrodán Undagoitia, ${ }^{12}$ J. Masbou, ${ }^{15}$ S. Mastroianni ${ }^{20}$ M. Messina ${ }^{18,8}$ K. Micheneau, ${ }^{15}$ K. Miller, ${ }^{19}$ A. Molinario, ${ }^{18}$ K. Morå, ${ }^{2}$ Y. Mosbacher, ${ }^{13}$ M. Murra, ${ }^{5}$ J. Naganoma, ${ }^{18,24}$

K. Ni, ${ }^{17}$ U. Oberlack, ${ }^{4}$ K. Odgers, ${ }^{10}$ J. Palacio, ${ }^{15}$ B. Pelssers, ${ }^{2}$ R. Peres, ${ }^{9}$ J. Pienaar, ${ }^{19}$ V. Pizzella, ${ }^{12}$ G. Plante, ${ }^{1}$ R. Podviianiuk, ${ }^{18}$ J. Qin, ${ }^{16}$ H. Qiu ${ }^{13}$ D. Ramírez García, ${ }^{14}$ S. Reichard, ${ }^{9,}$ B. Riedel, ${ }^{19}$ A. Rocchetti, ${ }^{14}$ N. Rupp, ${ }^{12}$ J. M. F. dos Santos, ${ }^{6}$ G. Sartorelli, ${ }^{3}$ N. Šarčević, ${ }^{14}$ M. Scheibelhut, ${ }^{4}$ S. Schindler, ${ }^{4}$ J. Schreiner, ${ }^{12}$ D. Schulte, ${ }^{5}$ M. Schumann, ${ }^{14}$ L. Scotto Lavina, ${ }^{22}$ M. Selvi, ${ }^{3}$ P. Shagin, ${ }^{24}$ E. Shockley,${ }^{19}$ M. Silva, ${ }^{6}$ H. Simgen, ${ }^{12}$ C. Therreau, ${ }^{15}$ D. Thers, ${ }^{15}$ F. Toschi, ${ }^{14}$ G. Trinchero, ${ }^{7}$ C. Tunnell,,${ }^{24}$ N. Upole, ${ }^{19}$ M. Vargas, ${ }^{5}$ G. Volta, ${ }^{9}$ O. Wack, ${ }^{12}$ H. Wang, ${ }^{25}$ Y. Wei, ${ }^{17}$ C. Weinheimer, ${ }^{5}$ D. Wenz, ${ }^{4}$ C. Wittweg, ${ }^{5}$ J. Wulf, ${ }^{9}$ J. Ye, ${ }^{17}$ Y. Zhang, ${ }^{1}$ T. Zhu, ${ }^{1}$ and J. P. Zopounidis ${ }^{22}$

$(\text { XENON Collaboration) })^{\|}$

\footnotetext{
${ }^{1}$ Physics Department, Columbia University, New York, New York 10027, USA

${ }^{2}$ Oskar Klein Centre, Department of Physics, Stockholm University, AlbaNova, Stockholm SE-10691, Sweden

${ }^{3}$ Department of Physics and Astronomy, University of Bologna and INFN-Bologna, 40126 Bologna, Italy

${ }^{4}$ Institut für Physik and Exzellenzcluster PRISMA, Johannes Gutenberg-Universität Mainz, 55099 Mainz, Germany

${ }^{5}$ Institut für Kernphysik, Westfälische Wilhelms-Universität Münster, 48149 Münster, Germany

${ }^{6}$ LIBPhys, Department of Physics, University of Coimbra, 3004-516 Coimbra, Portugal

${ }^{7}$ INAF-Astrophysical Observatory of Torino, Department of Physics, University of Torino and INFN-Torino, 10125 Torino, Italy

${ }^{8}$ New York University Abu Dhabi, PO Box 129188, Abu Dhabi, United Arab Emirates

${ }^{9}$ Physik-Institut, University of Zurich, 8057 Zurich, Switzerland

${ }^{10}$ Department of Physics, Applied Physics and Astronomy, Rensselaer Polytechnic Institute, Troy, New York 12180, USA

${ }^{11}$ Nikhef and the University of Amsterdam, Science Park, 1098XG Amsterdam, Netherlands

${ }^{12}$ Max-Planck-Institut für Kernphysik, 69117 Heidelberg, Germany

${ }^{13}$ Department of Particle Physics and Astrophysics, Weizmann Institute of Science, Rehovot 7610001, Israel

${ }^{14}$ Physikalisches Institut, Universität Freiburg, 79104 Freiburg, Germany

${ }^{15}$ SUBATECH, IMT Atlantique, CNRS/IN2P3, Université de Nantes, Nantes 44307, France

${ }^{16}$ Department of Physics and Astronomy, Purdue University, West Lafayette, Indiana 47907, USA

${ }^{17}$ Department of Physics, University of California, San Diego, California 92093, USA

${ }^{18}$ INFN-Laboratori Nazionali del Gran Sasso and Gran Sasso Science Institute, 67100 L'Aquila, Italy

${ }^{19}$ Department of Physics and Kavli Institute for Cosmological Physics, University of Chicago, Chicago, Illinois 60637, USA

${ }^{20}$ Department of Physics "Ettore Pancini," University of Napoli and INFN-Napoli, 80126 Napoli, Italy

${ }^{21}$ Kobayashi-Maskawa Institute for the Origin of Particles and the Universe, Nagoya University, Furo-cho, Chikusa-ku, Nagoya, Aichi 464-8602, Japan

${ }^{22}$ LPNHE, Université Pierre et Marie Curie, Université Paris Diderot, CNRS/IN2P3, Paris 75252, France

${ }^{23}$ LAL, Université Paris-Sud, CNRS/IN2P3, Université Paris-Saclay, F-91405 Orsay, France

${ }^{24}$ Department of Physics and Astronomy, Rice University, Houston, Texas 77005, USA

${ }^{25}$ Physics and Astronomy Department, University of California, Los Angeles, California 90095, USA
}

(Received 29 July 2019; revised manuscript received 7 November 2019; published 17 December 2019)

\begin{abstract}
Published by the American Physical Society under the terms of the Creative Commons Attribution 4.0 International license. Further distribution of this work must maintain attribution to the author(s) and the published article's title, journal citation, and DOI. Funded by $\operatorname{SCOAP}$.
\end{abstract}


We report constraints on light dark matter (DM) models using ionization signals in the XENON1T experiment. We mitigate backgrounds with strong event selections, rather than requiring a scintillation signal, leaving an effective exposure of (22 \pm 3 ) tonne day. Above $\sim 0.4 \mathrm{keV}_{e e}$, we observe $<1$ event/(tonne day $\mathrm{keV}_{e e}$ ), which is more than 1000 times lower than in similar searches with other detectors. Despite observing a higher rate at lower energies, no DM or CEvNS detection may be claimed because we cannot model all of our backgrounds. We thus exclude new regions in the parameter spaces for DM-nucleus scattering for DM masses $m_{\chi}$ within 3-6 GeV/c $c^{2}$, DM-electron scattering for $m_{\chi}>30 \mathrm{MeV} / c^{2}$, and absorption of dark photons and axionlike particles for $m_{\chi}$ within $0.186-1 \mathrm{keV} / c^{2}$.

DOI: 10.1103/PhysRevLett.123.251801

Introduction.-Substantial cosmological and astrophysical observations show that much of the Universe's mass consists of dark matter (DM) [1,2], and experiments aim to detect hypothetical DM particles and identify their nature $[3,4]$. The XENON1T experiment recently set the world's most stringent limits on DM-nucleus scattering for DM masses $m_{\chi} \geq 6 \mathrm{GeV} / c^{2} \quad[5,6]$. This Letter reanalyzes XENON1T's data to constrain lighter DM.

XENON1T [7] is a dual-phase time projection chamber (TPC) housed at the INFN Laboratori Nazionali del Gran Sasso. The active volume contains 2 tonne of liquid xenon (LXe) and is bounded by a grounded gate electrode at the top $(z=0)$ and a cathode at the bottom $(z=-97 \mathrm{~cm})$. Charged particles recoiling in LXe produce photons (scintillation) and electrons (ionization). XENON1T promptly detects the photons as the "S1" signal with 248 3-in. photomultiplier tubes (PMTs) positioned above and below the LXe target [8,9]. Electric fields drift the electrons upward and extract them into gaseous xenon, where electroluminescence produces a secondary scintillation "S2" signal. In most analyses, the ratio between S1 and $\mathrm{S} 2$ differentiates electronic recoils (ERs), caused by $\beta$ particles and $\gamma$ rays, from nuclear recoils (NRs), caused by neutrons or some DM particles. The interaction position, reconstructed from the S2 light pattern and the time difference between $\mathrm{S} 1$ and S2, discriminates DM candidates from most external radioactive backgrounds. XENON1T is shielded by a $3600 \mathrm{~m}$ water-equivalent rock overburden, an active water Cherenkov muon veto [10], and 1.2 tonne of LXe surrounding the TPC.

Dual-phase LXe TPCs are most sensitive to DM with masses $m_{\chi} \gtrsim 6 \mathrm{GeV} / c^{2}$, as lighter DM cannot transfer enough energy $(\sim 3.5 \mathrm{keV})$ to xenon nuclei to yield detectable S1's at a sufficient rate to be useful in DM experiments. S2's, however, are detectable in LXe at energies as low as $0.7 \mathrm{keV}$ for nuclear recoils and $0.186 \mathrm{keV}$ for electronic recoils $[11,12]$. Here, we reanalyze XENON1T's data without requiring an S1-an "S2only analysis." As in previous S2-only analyses [13,14], substantial backgrounds preclude detection claims. However, we use strong event selections to reduce these backgrounds considerably and subtract some known background components.

Data selection.-We use the main science run (SR1) of XENON1T $[5,6]$ with a live time of 258.2 days, after excluding time when the data acquisition was insensitive, the muon veto fired, or a PMT showed excessive pulse rates [7]. Reference [5] derived a $~ 4 \%$ shorter live time because it excluded time just after high-energy events. Backgrounds from these periods are mitigated by other methods here.

We used $30 \%$ of SR1 events as training data, distributed homogeneously in time, to determine event selections and, for each dark matter model and mass, a region of interest (ROI) in terms of the integrated S2 charge ("S2 size"). Limits on DM parameters are computed using only the remaining $70 \%$ (the search data, 180.7 days), which was not examined with the strong event selections used here until the analysis was fixed. We chose selections to remove large identifiable background populations, but retain the central part of the DM signal model in different observable dimensions. We use a single set of selections; only the S2 ROIs vary for different DM models and masses.

We determine the efficiency of our selections in the 2D space of (uncorrected) S2 signal size and interaction depth $z$-since our signal models vary strongly along both dimensions - using calibration data and simulated waveforms [15]. Figure 1 shows the effective remaining search data exposure after selections. Figure 2 shows the efficiencies of the most impactful cuts in the most important

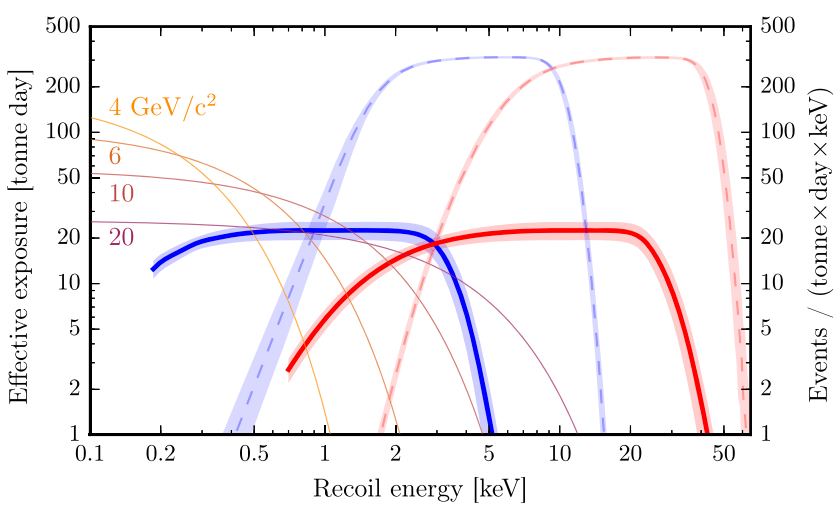

FIG. 1. Effective remaining exposure after event selections for NR (red) and ER (blue) signals of different energies, for $\mathrm{S} 2 \in[150,3000] \mathrm{PE}$, on the left $y$ axis. Dashed lines show the same for XENON1T's main analysis [5], and shaded bands show $\pm 1 \sigma$ systematic uncertainties. Thin lines show the expected differential event rate for $4,6,10$, and $20 \mathrm{GeV} / c^{2}$ spin-independent (SI) DM-nucleus scattering with $\sigma=10^{-43} \mathrm{~cm}^{2}$, under the nominal signal model, on the right $y$ axis. 


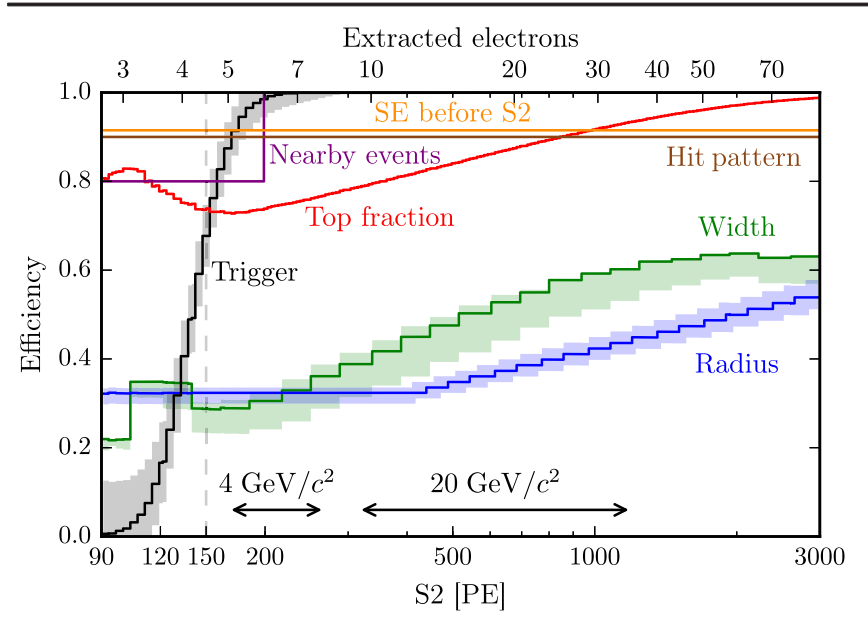

FIG. 2. Efficiencies (fraction of signal events passed) of the most impactful event selections vs S2 size for $z \in[-30,-10] \mathrm{cm}$, applicable if a given selection is applied last. Solid lines correspond to the nominal detector response model, bands to $\pm 1 \sigma$ variations of model parameters. The arrows show the S2 ROIs for the 4 and $20 \mathrm{GeV} / c^{2}$ spin-independent NR DM analyses. Events below $150 \mathrm{PE}$ are not used but shown for completeness. The top horizontal axis shows the approximate number of extracted electrons corresponding to each S2 size. The combined efficiency of the selections not shown here is $\sim 93 \%$.

$z$ range for light DM. XENON1T's trigger efficiency, shown in black in Fig. 2, is determined as in Ref. [16]. Events with S2 below 150 photoelectrons (PE), i.e. 4.5 extracted electrons, are not used, but they are shown for completeness. Previous XENON1T analyses [5,6,17,18] applied a similar threshold of $200 \mathrm{PE}$; the S1 requirement, not the S2 threshold, limited their light DM sensitivity.

Without S1's, the event depth $z$ cannot be accurately estimated. However, the S2 waveform width in time is correlated with $z$, due to diffusion of the electrons during drift [19]. Rather than estimating and constraining $z$, we remove events with S2 width outside [835, 1135] ns, as shown in Fig. 3. This window has a high expected signal rate, but showed few events in the training data, indicating a low background. The width cut mitigates backgrounds with atypically wide S2's, consistent with $\beta$ decays occurring on the cathode wires. The field geometry there causes charge loss, which in turn causes the cathode events to have unusually small S2's for their energy. Many have detectable S1's and are called "S1-tagged cathode events"; these are easily removed by another cut (described below). The width cut also mitigates backgrounds with atypically narrow S2's, which could similarly result from decays on the electrodes at the top of the TPC.

The width cut efficiency, calculated with simulated S2 waveforms, is shown in green in Fig. 2. The simulated waveform's median widths agree to within $\sim 50$ ns with those observed in deuterium-deuterium plasma fusion neutron generator calibration data [20], as detailed in Supplemental Material [21]. The cut efficiency is highest

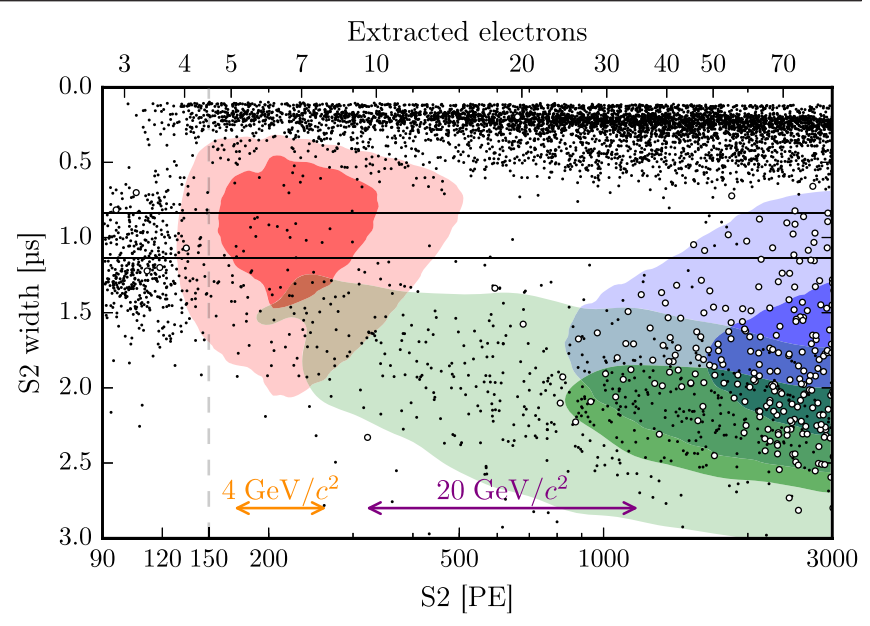

FIG. 3. Observed events in the search data. Events between the horizontal black lines pass all cuts, the others fail only the cut on S2 width. Open circles demark events with S1's, dots events without. The colored regions contain 50\% (faint, 90\%) of expected events from the three background components described in the text: flat-spectrum ER (blue), CEvNS (red), and cathode events (green). The arrows denote two S2 ROIs and the dashed line the S2 threshold, as in Fig. 2.

in the $z \in[-30,-10] \mathrm{cm}$ range presented in Fig. 2, where the expected DM signals are most distinguishable from backgrounds. We include a $\pm 50 \mathrm{~ns}$ systematic uncertainty on the S2 width, creating a 12\% uncertainty in the expected $4 \mathrm{GeV} / c^{2}$ spin-independent (SI) NR DM event rate.

We remove events reconstructed at high radii $R$. The threshold is $R^{2}=700 \mathrm{~cm}^{2}$ for $\mathrm{S} 2 \leq 400 \mathrm{PE}$ and then rises linearly with $\log (\mathrm{S} 2)$ to $1150 \mathrm{~cm}^{2}$ at $3000 \mathrm{PE}$. As described in Ref. [22], events on the TPC wall $(R=47.9 \mathrm{~cm})$ have unusually small S2's because electrons are lost on the TPC walls. They can be misreconstructed inward due to the increased position reconstruction uncertainty for small S2's $(1.8 \mathrm{~cm}$ at $\mathrm{S} 2=200 \mathrm{PE})$ and, more importantly, the inhomogeneous drift field. The latter cannot be mitigated as in Ref. [22] because S1's are needed to estimate $z$ reliably. The efficiency of the radial cut, shown in blue in Fig. 2, is estimated with ${ }^{83 m} \mathrm{Kr}$ calibration data. We introduce an uncertainty to bracket variations in the estimate from ${ }^{83 m} \mathrm{Kr}$ datasets at different times and in a simple geometric calculation. This introduces an $8 \%$ uncertainty in the $4 \mathrm{GeV} / c^{2}$ spin-independent NR DM rate.

On average, $\sim 63 \%$ of S2 light is seen by the top PMT array, with a $\sim 3 \%$ position-dependent variation for which we correct. We remove events in which this fraction is $>66 \%$, indicative of events produced in the gaseous xenon phase above the usual secondary scintillation region ("gas events"). The efficiency, shown in red in Fig. 2, is calculated from binomial fluctuations in photon detection and a small intrinsic spread measured at high S2. We verified that the resulting efficiency is conservative using neutron generator data. The efficiency rises below $\sim 170 \mathrm{PE}$ 
as the trigger preselects S2's to which many PMTs contribute, which is rarer for S2's seen mostly by the top array.

Pileup of randomly emitted single-electron (SE) signals can be misidentified as S2's from real events. We employ three cuts against this background, without which the population most prominent at $\lesssim 150 \mathrm{PE}$ in Fig. 3 would be $\sim 50 \times$ larger. First, we remove events whose S2 hit pattern on the top array is inconsistent with that of single scatters, as determined by a likelihood test [15]. This cut has a 90\% efficiency, shown in brown in Fig. 2, as measured with neutron generator data and S1-tagged cathode events. This cut also removes some unresolved double scatter events, e.g., from radiogenic neutrons. Second, we exclude events with one or more S2 or single-electron signals up to $\sim 1 \mathrm{~ms}$ before the largest S2, with $91.5 \%$ efficiency, as measured with high-energy background events and shown in orange in Fig. 2. This cut also suppresses gas events, whose S1's are broader than those of events in the liquid and therefore often misidentified as S2's. Third, as high-energy events cause a temporary and localized enhancement in single-electron emission [23], we utilize a combined $p$-value cut [24] against events close in time or reconstructed position to recent highenergy events, with $80 \%$ efficiency, as determined with S1-tagged cathode events and shown in purple in Fig. 2. This last cut only helps against the single-electron pileup background, so we apply it only for S2 $<200 \mathrm{PE}$.

We exclude events in which the S2 waveform is distorted by a merged $\mathrm{S} 1$, with $~ 95 \%$ efficiency, as determined with ${ }^{220} \mathrm{Rn}[25]$ and neutron generator data. To remove double scatters, we apply the same cut to events with substantial secondary S2's as in Refs. [5,15], with $99.5 \%$ efficiency.

Finally, we apply two cuts specifically to events with S1's. Events whose drift time indicates a $z$ outside $[-95,-7] \mathrm{cm}$ are removed, to exclude events high in the detector and S1-tagged cathode events. We assume no signal or background events are produced outside this $z$ region. Our assumption is conservative because this is a limit-only analysis. We also remove events with a very large S1 (> $200 \mathrm{PE}$ ), with negligible efficiency loss.

Detector response.-We compute XENON1T's response to ERs and NRs in the same two-dimensional $(\mathrm{S} 2, z)$ space used for the efficiencies and project the model after applying efficiencies onto S2 for comparison with data. We use the best-fit detector response model from Ref. [22], but we assume in our signal and background models that NRs below $0.7 \mathrm{keV}$ and ERs below $186 \mathrm{eV}$ ( $\sim 12$ produced electrons) are undetectable, as the LXe charge yield $Q_{y}$ has never been measured below these energies. Even without these cutoffs, the low-energy $Q_{y}$ from Ref. [22] is lower than that favored by other LXe measurements [11,12] and models [26]. Thus, our results should be considered conservative.

While a complete model of backgrounds in the S2-only channel is unavailable, we can quantify three components

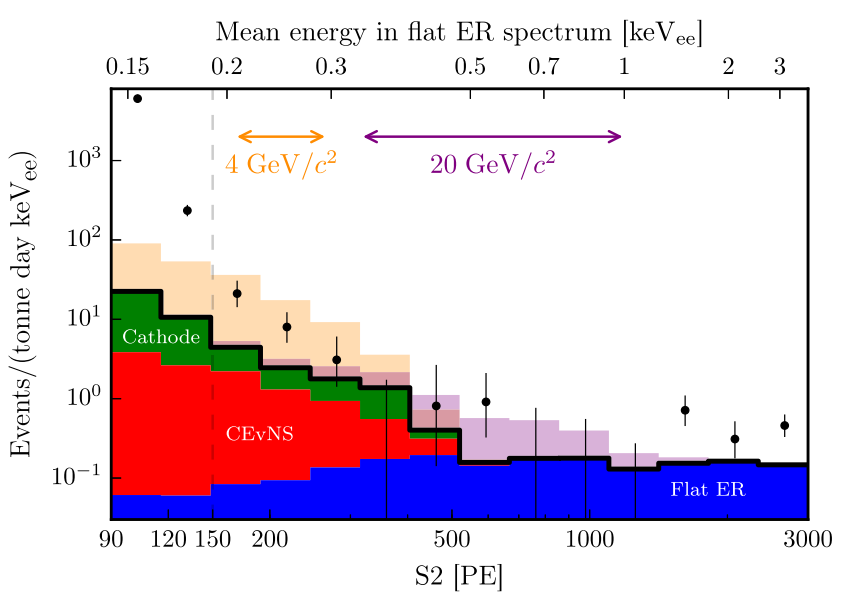

FIG. 4. Distribution of events that pass all cuts (black dots); error bars show statistical uncertainties ( $1 \sigma$ Poisson). The thick black line shows the predetermined summed background model, below which its three components are indicated, with colors as in Fig. 3. The lightly shaded orange (purple) histogram, stacked on the total background, shows the signal model for $4 \mathrm{GeV} / c^{2}$ $\left(20 \mathrm{GeV} / c^{2}\right)$ SI DM models excluded at exactly $90 \%$ confidence level. The arrows show the ROIs for these analyses, and the dashed line the S2 threshold, as in Figs. 2 and 3. All rates are shown relative to the effective remaining exposure after selections. The top $x$ axis shows the mean expected energy of events after cuts for a flat ER spectrum if there were no $Q_{y}$ cutoff.

of the background, illustrated in Figs. 3 and 4. First, the ER background from high $Q$-value $\beta$ decays, primarily ${ }^{214} \mathrm{~Pb}$ $(Q=1.02 \mathrm{MeV})$ [22], is flat in our energy range of interest. We use a rate of 0.142 events/(tonne day $\mathrm{keV}$ ), a conservative lower bound derived from $<210 \mathrm{keV}$ data. Second, coherent nuclear scattering of ${ }^{8} \mathrm{~B}$ solar neutrinos (CEvNS), shown in red in Fig. 3, should produce a background nearly identical to a $6 \mathrm{GeV} / c^{2}, 4 \times$ $10^{-45} \mathrm{~cm}^{2}$ spin-independent NR DM signal [27,28]. We expect $2.0 \pm 0.3 \mathrm{CEvNS}$ events inside the $6 \mathrm{GeV} / c^{2}$ SI NR ROI. Third, we see events from $\beta$ decays on the cathode wires. Sufficiently low-energy cathode events lack S1's. We derive a lower bound on this background using the ratio of events with and without S1's measured in a high-S2, high width control region where cathode events are dominant. This procedure is detailed in Supplemental Material [21].

Figure 4 compares the observed events to our nominal signal and background models. For $\mathrm{S} 2 \gtrsim$ $300 \mathrm{PE}\left(\sim 0.3 \mathrm{keV}_{e e}\right)$, we observe rates well below $1 /\left(\right.$ tonne day $\left.\mathrm{keV}_{e e}\right)$, more than 1000 times lower than previous S2-only analyses [14,29]. Below $150 \mathrm{PE}$, the rate rises quickly, likely due to unmodeled backgrounds.

DM models.-We constrain several DM models, using Ref. [30] to compute the energy spectra. First, we consider spin-independent and spin-dependent (SD) DM-nucleus scattering with the same astrophysical $\left(v_{0}, v_{\text {esc }}\right.$, etc.) and particle physics models (form factors, structure functions) as Refs. [5,6]. For SD scattering, we consider the neutrononly (to first order) coupling specifically. If the DM-matter 
interaction is mediated by a (scalar) particle of mass $m_{\phi}$, the differential rate has a factor $m_{\phi}^{4} /\left(m_{\phi}^{2}+q^{2} / c^{2}\right)^{2}$, with $q=$ $\sqrt{2 m_{N} E_{R}}$ the momentum transfer, $E_{R}$ the recoil energy, and $m_{N}$ the nuclear mass [31-33]. Usually, this factor is considered to be $\sim 1$, corresponding to $m_{\phi} \gtrsim$ $100 \mathrm{MeV} / c^{2}$. We also consider the SI light-mediator limit, $m_{\phi} \ll q / c \approx 10^{-3} m_{\chi}$ (for $m_{\chi} \ll m_{N}$ ), in which the differential event rate for DM-nucleus scattering scales with $m_{\phi}^{4}$.

Second, light DM could be detected from its scattering off bound electrons. We follow Ref. [34] to calculate the
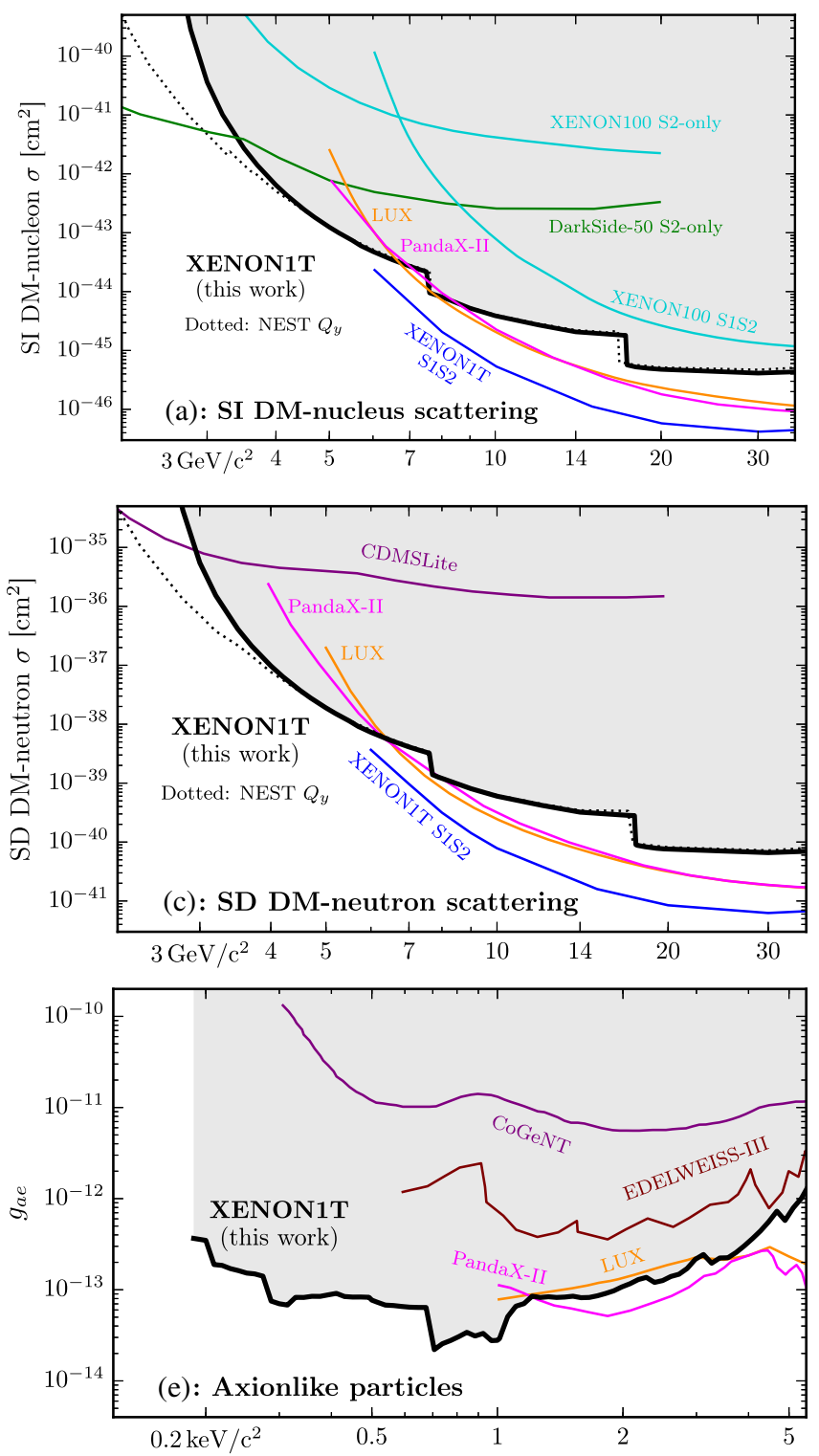

DM-electron scattering rates, using the ionization form factors from Ref. [35], the detector response model as above (from Ref. [22]), and dark matter form factor 1 . Relativistic calculations [36] predict 2-10 times larger rates (for $\geq 5$ produced electrons), and thus our results should be considered conservative. As previous DM-electron scattering results $[34,37,38]$ did not use a $Q_{y}$ cutoff, we derive constraints with and without signals below 12 produced electrons (equivalent to our $Q_{y}$ cutoff) to ease comparison.
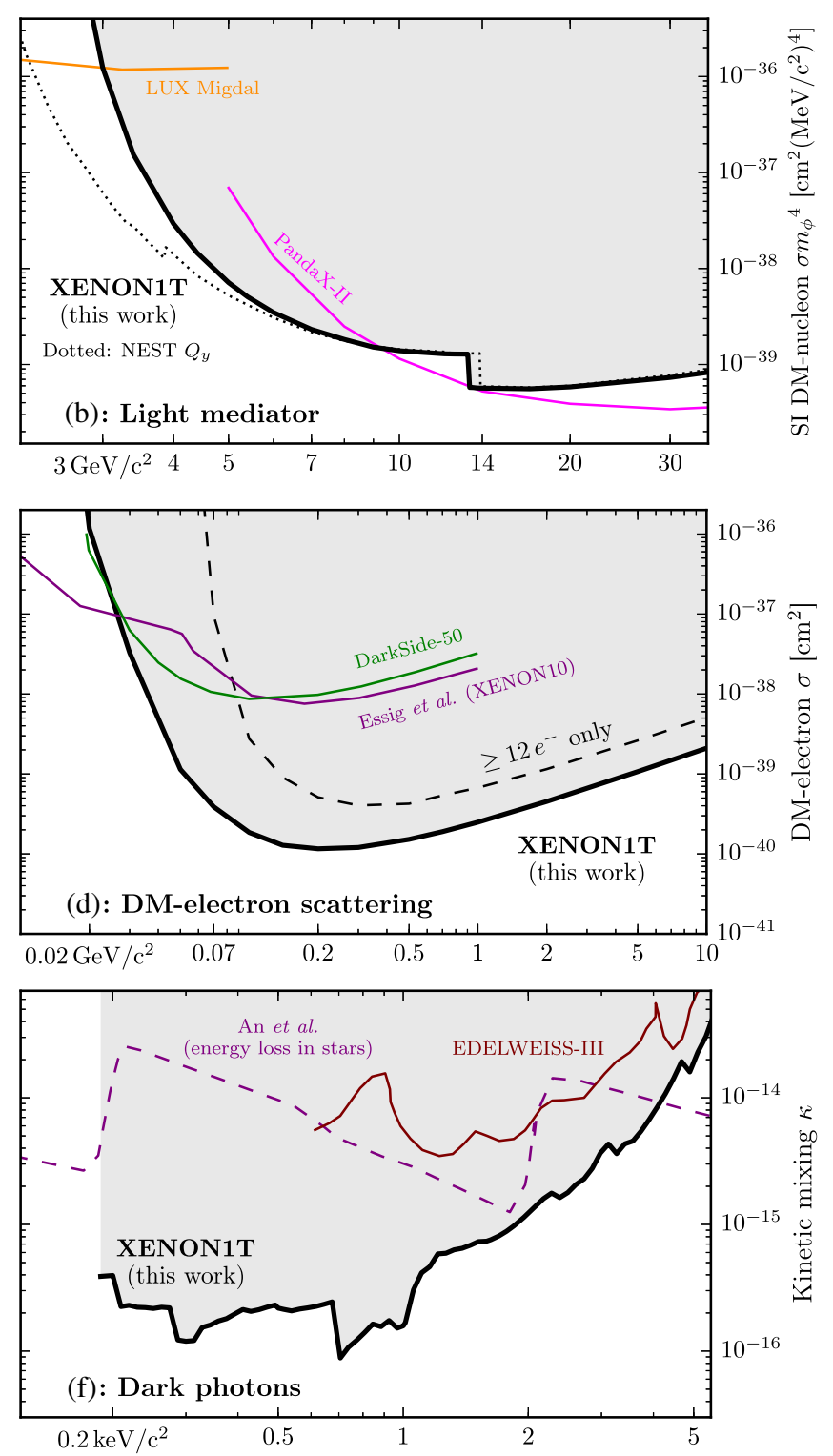

FIG. 5. The 90\% confidence level upper limits (black lines with gray shading above) on DM-matter scattering for the models discussed in the text, with the dark matter mass $m_{\chi}$ on the horizontal axes. We show other results from XENON1T in blue [5,6], LUX in orange [45-48], PandaX-II in magenta [33,49,50], DarkSide-50 in green [29,38,51], XENON100 in turquoise [14,52], EDELWEISS-III [53] in maroon, and other constraints [34,54-56] in purple. Dotted lines in (a)-(c) show our limits when assuming the $Q_{y}$ from NEST v2.0.1 [42] cut off below $0.3 \mathrm{keV}$. The dashed line in (d) shows the limit without considering signals with < 12 produced electrons; the solid line can be compared to the constraints from Refs. [34,38] shown in the same panel, the dashed line to our results on other DM models, which use the $Q_{y}$ cutoffs described in the text. The limits jump at $17.5 \mathrm{GeV} / c^{2}$ in (a) (and similarly elsewhere) because the observed count changes from 10 to 3 events in the ROIs left and right of the jump, respectively. 
Third, bosonic DM candidates, such as dark photons and axionlike particles, can be absorbed by xenon atoms, analogous to photons in the photoelectric effect. The result is a monoenergetic ER signal at $E_{\chi}=m_{\chi} c^{2}$, with rates of

$$
\left[\begin{array}{c}
4 \times 10^{23} \mathrm{keV}^{2} / E_{\chi} \\
1.3 \times 10^{19} \mathrm{keV}^{-1} g_{\mathrm{ae}}^{2} E_{\chi}
\end{array}\right] \frac{\sigma_{\mathrm{pe}}}{A} \mathrm{~kg}^{-1} \mathrm{day}^{-1},
$$

where the top row corresponds to dark photons [39] and the bottom to axionlike particles [40]. Here $\sigma_{\mathrm{pe}}$ is xenon's photoelectric cross section at $E_{\chi}$ in barn, $A$ xenon's mean atomic mass number, $\kappa$ the dark photon-photon kinetic mixing parameter, and $g_{\text {ae }}$ the axioelectric coupling constant. This process allows us to constrain $\mathrm{keV}$-scale DM candidates.

Inference and results.-We constrain these DM models based on the number of events in predetermined S2 ROIs, which vary for each model and mass. The ROIs are optimized to give stringent limits on the training data, while requiring that the lower (upper) bound is between the fifth and 60th (40th and 95th) percentile of the signal distribution in $\mathrm{S} 2 \in[90,3000] \mathrm{PE}$ after selections, and never below $150 \mathrm{PE}$. These constraints, the event selections, and the background models were set before examining the search data. Because of the finite training data, the ROI bounds are nonsmooth functions of DM mass.

We compute an aggregate uncertainty on the signal and background expectations in the ROIs, including the $\sim 5 \%$ uncertainty on electron lifetime and $\sim 2.5 \%$ uncertainty on the S2 gain $g_{2}$, besides the (more impactful) uncertainties on the efficiencies mentioned above. We then compute 90\% confidence level upper limits using the standard Poisson method [41]. To ensure the limits are conservative (statistically overcover), we use the tenth percentile signal and background expectations-i.e., the nominal value minus $\sim 1.28 \times$ the aggregate uncertainty-and never exclude signals with $<2.3$ expected events.

The resulting DM upper limits are shown in Fig. 5. We exclude new regions in the parameter spaces for all DM models shown. ER models [Figs. 5(d)-5(f)] benefit most from an S2-only analysis, as ERs produce smaller S1's than NRs at the same S2 size. Our constraints on $6 \mathrm{GeV} / c^{2} \mathrm{SI}$ NR DM, and therefore ${ }^{8} \mathrm{~B}$ CEvNS, are weaker than in Ref. [5] due to the 16 observed events in the $6 \mathrm{GeV} / c^{2}$ ROI and an underfluctuation of the background in Ref. [5]. Dotted lines in Figs. 5(a)-5(c) show our constraints given the NR $Q_{y}$ from NEST v2.0.1 [42] cut off below $0.3 \mathrm{keV}$, which accommodates a measurement [43] released shortly after our analysis was completed. Future S2-only studies can improve on these results using next-generation detectors such as XENONnT and LZ [44], lower-energy calibrations, and additional mitigation of backgrounds.

We thank Tien-Tien Yu for helpful correspondence on the DM-electron scattering form factors. We gratefully acknowledge support from the National Science
Foundation, Swiss National Science Foundation, German Ministry for Education and Research, Max Planck Gesellschaft, Deutsche Forschungsgemeinschaft, Netherlands Organisation for Scientific Research (NWO), Netherlands eScience Center (NLeSC) with the support of the SURF Cooperative, Weizmann Institute of Science, Israeli Centers of Research Excellence (I-CORE), Pazy-Vatat, Fundacao para a Ciencia e a Tecnologia, Region des Pays de la Loire, Knut and Alice Wallenberg Foundation, Kavli Foundation, and Istituto Nazionale di Fisica Nucleare. This project has received funding or support from the European Union's Horizon 2020 research and innovation programme under the Marie SklodowskaCurie Grant Agreements No. 690575 and No. 674896, respectively. Data processing is performed using infrastructures from the Open Science Grid and European Grid Initiative. We are grateful to Laboratori Nazionali del Gran Sasso for hosting and supporting the XENON project.

*jelle.aalbers@fysik.su.se

Also at: Institute for Subatomic Physics, Utrecht University, Utrecht, Netherlands.

*Also at: Coimbra Polytechnic-ISEC, Coimbra, Portugal.

§shayne@physik.uzh.ch

"xenon@lngs.infn.it

[1] G. Bertone, D. Hooper, and J. Silk, Particle dark matter: evidence, candidates and constraints, Phys. Rep. 405, 279 (2005).

[2] P. A. R. Ade et al. (Planck Collaboration), Planck 2018 results. VI. Cosmological parameters, arXiv:1807.06209.

[3] L. Roszkowski, E. M. Sessolo, and S. Trojanowski, WIMP dark matter candidates and searches-current status and future prospects, Rep. Prog. Phys. 81, 066201 (2018).

[4] T. Marrodán Undagoitia and L. Rauch, Dark matter directdetection experiments, J. Phys. G 43, 013001 (2016).

[5] E. Aprile et al. (XENON Collaboration), Dark Matter Search Results from a One Ton-Year Exposure of XENON1T, Phys. Rev. Lett. 121, 111302 (2018).

[6] E. Aprile et al. (XENON Collaboration), Constraining the Spin-Dependent WIMP-Nucleon Cross Sections with XENON1T, Phys. Rev. Lett. 122, 141301 (2019).

[7] E. Aprile et al. (XENON Collaboration), The XENON1T dark matter experiment, Eur. Phys. J. C 77, 881 (2017).

[8] E. Aprile et al. (XENON Collaboration), Lowering the radioactivity of the photomultiplier tubes for the XENON1T dark matter experiment, Eur. Phys. J. C 75, 546 (2015).

[9] P. Barrow et al. Qualification tests of the R11410-21 photomultiplier tubes for the XENON1T detector, J. Instrum. 12, P01024 (2017).

[10] E. Aprile et al. (XENON Collaboration), Conceptual design and simulation of a water Cherenkov muon veto for the XENON1T experiment, J. Instrum. 9, P11006 (2014).

[11] D. S. Akerib et al. (LUX Collaboration), Low-energy (0.7$74 \mathrm{keV}$ ) nuclear recoil calibration of the LUX dark matter experiment using D-D neutron scattering kinematics, arXiv:1608.05381. 
[12] D. S. Akerib et al. (LUX Collaboration), Ultra-Low Energy Calibration of LUX Detector using ${ }^{127} \mathrm{Xe}$ Electron Capture, Phys. Rev. D 96, 112011 (2017).

[13] J. Angle et al. (XENON10 Collaboration), Search for Light Dark Matter in XENON10 Data, Phys. Rev. Lett. 107, 051301 (2011); 110, 249901(E) (2013).

[14] E. Aprile et al. (XENON100 Collaboration), Low-mass dark matter search using ionization signals in XENON100, Phys. Rev. D 94, 092001 (2016); 95, 059901(E) (2017).

[15] E. Aprile et al. (XENON Collaboration), XENON1T dark matter data analysis: signal reconstruction, calibration and event selection, Phys. Rev. D 100, 052014 (2019).

[16] E. Aprile et al. (XENON Collaboration), The XENON1T Data Acquisition System, JINST 14, P07016 (2019).

[17] E. Aprile et al. (XENON Collaboration), First Results on the Scalar WIMP-Pion Coupling, Using the XENON1T Experiment, Phys. Rev. Lett. 122, 071301 (2019).

[18] E. Aprile et al. (XENON Collaboration), First Dark Matter Search Results from the XENON1T Experiment, Phys. Rev. Lett. 119, 181301 (2017).

[19] P. Sorensen, Anisotropic diffusion of electrons in liquid xenon with application to improving the sensitivity of direct dark matter searches, Nucl. Instrum. Methods Phys. Res., Sect. A 635, 41 (2011).

[20] R. F. Lang, J. Pienaar, E. Hogenbirk, D. Masson, R. Nolte, A. Zimbal, S. Röttger, M. L. Benabderrahmane, and G. Bruno, Characterization of a deuterium-deuterium plasma fusion neutron generator, Nucl. Instrum. Methods Phys. Res., Sect. A 879, 31 (2018).

[21] See Supplemental Material at http://link.aps.org/ supplemental/10.1103/PhysRevLett.123.251801 for numerical results for particular dark matter models and masses, as well as quantitative details on the energy scale, S2 width model and cathode background estimate.

[22] E. Aprile et al. (XENON Collaboration), XENON1T dark matter data analysis: Signal and background models and statistical inference, Phys. Rev. D 99, 112009 (2019).

[23] P. Sorensen and K. Kamdin, Two distinct components of the delayed single electron noise in liquid xenon emission detectors, J. Instrum. 13, P02032 (2017).

[24] S. A. Stouffer et al., The American Soldier: Adjustment during Army Life, Vol. 1 (Princeton University Press, Princeton, NJ, 1949).

[25] E. Aprile et al. (XENON Collaboration), Results from a calibration of XENON100 using a source of dissolved radon-220, Phys. Rev. D 95, 072008 (2017).

[26] M. Szydagis et al., Noble element simulation technique v2.0, 2018, https://doi.org/10.5281/zenodo.1314669.

[27] D. Akimov et al. (COHERENT Collaboration), Observation of coherent elastic neutrino-nucleus scattering, Science 357, 1123 (2017).

[28] A. M. Serenelli, W. C. Haxton, and C. Peña-Garay, Solar models with accretion. I. Application to the solar abundance problem, Astrophys. J. 743, 24 (2011).

[29] P. Agnes et al. (DarkSide Collaboration), Low-Mass Dark Matter Search with the DarkSide-50 Experiment, Phys. Rev. Lett. 121, 081307 (2018).

[30] J. Aalbers, B. Pelssers, and K. Morå, Wimprates v0.3.0, 2019, http://doi.org/10.5281/zenodo.3345959.
[31] N. Fornengo, P. Panci, and M. Regis, Long-range forces in direct dark matter searches, Phys. Rev. D 84, 115002 (2011).

[32] E. Del Nobile, M. Kaplinghat, and H.-B. Yu, Direct detection signatures of self-interacting dark matter with a light mediator, J. Cosmol. Astropart. Phys. 10 (2015) 055.

[33] X. Ren et al. (PandaX-II Collaboration), Constraining Dark Matter Models with a Light Mediator at the PandaX-II Experiment, Phys. Rev. Lett. 121, 021304 (2018).

[34] R. Essig, T. Volansky, and T.-T. Yu, New constraints and prospects for sub-GeV dark matter scattering off electrons in xenon, Phys. Rev. D 96, 043017 (2017).

[35] T.-T. Yu, Direct detection of sub-GeV dark matter, http:// ddldm.physics.sunysb.edu/ddlDM.

[36] M. K. Pandey et al., Constraints on spin-independent dark matter scattering off electrons with germanium and xenon detectors, arXiv:1812.11759.

[37] R. Essig, A. Manalaysay, J. Mardon, P. Sorensen, and T. Volansky, First Direct Detection Limits on Sub-GeV Dark Matter from XENON10, Phys. Rev. Lett. 109, 021301 (2012).

[38] P. Agnes et al. (DarkSide Collaboration), Constraints on Sub-GeV Dark-Matter-Electron Scattering from the DarkSide-50 Experiment, Phys. Rev. Lett. 121, 111303 (2018).

[39] M. Pospelov, A. Ritz, and M. Voloshin, Bosonic superWIMPs as keV-scale dark matter, Phys. Rev. D 78, 115012 (2008).

[40] K. Arisaka, P. Beltrame, C. Ghag, J. Kaidi, K. Lung, A. Lyashenko, R. D. Peccei, P. Smith, and K. Ye, Expected sensitivity to galactic/solar axions and bosonic superWIMPs based on the axio-electric effect in liquid xenon dark matter detectors, Astropart. Phys. 44, 59 (2013).

[41] M. Tanabashi et al. (Particle Data Group), Review of particle physics, Phys. Rev. D 98, 030001 (2018).

[42] M. Szydagis et al., Noble element simulation technique v2.0.1, 2019, https://doi.org/10.5281/zenodo.3357973.

[43] B. Lenardo et al., Measurement of the ionization yield from nuclear recoils in liquid xenon between $0.3-6 \mathrm{keV}$ with single-ionization-electron sensitivity, arXiv:1908.00518.

[44] B. J. Mount et al. (LZ Collaboration), LUX-ZEPLIN (LZ) technical design report, arXiv:1703.09144.

[45] D. S. Akerib et al. (LUX Collaboration), Results from a Search for Dark Matter in the Complete LUX Exposure, Phys. Rev. Lett. 118, 021303 (2017).

[46] D. S. Akerib et al. (LUX Collaboration), Results of a Search for Sub-GeV Dark Matter Using 2013 LUX Data, Phys. Rev. Lett. 122, 131301 (2019).

[47] D. S. Akerib et al. (LUX Collaboration), Limits on SpinDependent WIMP-Nucleon Cross Section Obtained from the Complete LUX Exposure, Phys. Rev. Lett. 118, 251302 (2017).

[48] D. S. Akerib et al. (LUX Collaboration), First Searches for Axions and Axionlike Particles with the LUX Experiment, Phys. Rev. Lett. 118, 261301 (2017).

[49] C. Fu et al. (PandaX-II Collaboration), Limits on Axion Couplings from the First 80 Days of Data of the PandaX-II Experiment, Phys. Rev. Lett. 119, 181806 (2017).

[50] X. Cui et al. (PandaX-II Collaboration), Dark Matter Results from 54-Ton-Day Exposure of PandaX-II Experiment, Phys. Rev. Lett. 119, 181302 (2017). 
[51] P. Agnes et al. (DarkSide Collaboration), DarkSide-50 532day dark matter search with low-radioactivity argon, Phys. Rev. D 98, 102006 (2018).

[52] E. Aprile et al. (XENON100 Collaboration), XENON100 dark matter results from a combination of 477 live days, Phys. Rev. D 94, 122001 (2016).

[53] E. Armengaud et al. (EDELWEISS Collaboration), Searches for electron interactions induced by new physics in the EDELWEISS-III germanium bolometers, Phys. Rev. D 98, 082004 (2018).
[54] C. E. Aalseth et al. (CoGeNT Collaboration), Experimental Constraints on a Dark Matter Origin for the DAMA Annual Modulation Effect, Phys. Rev. Lett. 101, 251301 (2008); 102, 109903(E) (2009).

[55] R. Agnese et al. (SuperCDMS Collaboration), Low-mass dark matter search with CDMSlite, Phys. Rev. D 97, 022002 (2018).

[56] H. An, M. Pospelov, J. Pradler, and A. Rit, Direct detection constraints on dark photon dark matter, Phys. Lett. B 747, 331 (2015). 